\title{
Survival Prediction in Gliomas: Current State and Novel Approaches
}

\author{
Rachel Zhao • Andra Valentina Krauze \\ Faculty of Medicine, University of British Columbia, Vancouver, Canada
}

Author for Correspondence: Andra Valentina Krauze, Division of Radiation Oncology and Developmental Radiotherapeutics, BC Cancer Surrey, University of British Columbia, Vancouver, Canada. Email: andra.krauze@bccancer.bc.ca

Doi: https://doi.org/10.36255/exonpublications.gliomas.2021.chapter9

\begin{abstract}
Gliomas are neurologically devastating tumors with generally poor outcomes. Traditionally, survival prediction in glioma is studied from clinical features using statistical approaches. With the rapid development of artificial intelligence approaches encompassing machine learning and deep learning, there has been a keen interest among researchers to apply these methods to survival prediction in glioma allowing for integrated processes that encompass pathology, histology, molecular, imaging, and clinical features. This chapter provides an overview of the emerging computational approaches that have the potential to revolutionize survival prediction in glioma. Machine learning and deep learning techniques, including support vector machine, random forest, convolutional neural network, and radiomics, are discussed.
\end{abstract}

Keywords: convolutional neural network; machine learning approaches; support vector machine; radiomics; survival prediction in glioma

In: Gliomas. Debinski W (Editor). Exon Publications, Brisbane, Australia. ISBN: 978-0-6450017-4-7; Doi: https://doi.org/10.36255/exonpublications.gliomas.2021

Copyright: The Authors.

License: This open access article is licenced under Creative Commons Attribution-NonCommercial 4.0 International (CC BY-NC 4.0) https://creativecommons.org/licenses/by-nc/4.0/ 


\section{INTRODUCTION}

Outcome prediction in glioma is of tremendous importance as it has the potential to aid in optimal patient management and patient counselling. In the past, outcome prediction has centered mainly on clinical features such as age and performance status, surgical features such as resection status, and pathological features such as morphology and Word Health Organization (WHO) grade. Molecular classification and identification of predictive and prognostic factors are now also considered (1-4). Traditionally, outcome prediction is carried out through recursive partitioning analysis (RPA) $(3,4)$. Glioblastoma patient survival by prognostic grouping was published initially in 1993 and employed RPA, a non-parametric statistical technique creating prognostic groups based on clinical features (3). It divided patients into six prognostic classes (I - VI) (3), later simplified to three classes (III, IV, and V/VI) and eventually revised to include only glioblastoma (4). Limitations included the lack of temozolomide-based stratification and molecular features (for example, $\mathrm{O}^{6}$-Methylguanine-DNA-methyl transferase [MGMT] methylation), both rectified recently $(1,2)$.

In the clinic, the most debated scenarios regarding management and outcomes center on the elderly (5-10), patients with lower grade gliomas (11-14), and the administration of systemic therapy (for example, PCV [Procarbazine, CCNU and vincristine] vs. temozolomide, current vs. sequential, and number of cycles) (15-17). With respect to glioma in the elderly, a number of metrics including age (6), temporal muscle thickness (7) and geriatric assessment (8) have been employed to predict survival. Straube et al. created a scoring system incorporating age, performance status, MGMT status, the extent of resection, and aphasia and motor dysfunction after surgery, all of which were found to be associated with survival (10). In lower grade gliomas, current practice is based on old pathological classification of glioma (12). This has resulted in the lack of a consensus regarding optimal use of radiotherapy in patients with low grade glioma (LGG) (13), timing and dose of radiation, and timing of chemotherapy (14). For survival prediction, consensus is generally achieved by multidisciplinary review of histology, molecular and imaging factors. Gittleman et al. employed TCGA (The Cancer Genome Atlas) data to develop and externally validate a survival nomogram, which is available as a free online software, for lower-grade glioma patients. The final nomogram included factors that increased the probability of survival: grade II tumor, younger age at diagnosis, a higher KPS (Karnosfsky Performance Status) and IDH (isocitrate dehydrogenase) mutation (16). In high-grade glioma (HGG) including glioblastoma, there is ongoing controversy regarding the number of cycles of chemotherapy to be administered (15-17). Gittleman et al. developed a nomogram using the Cox proportional hazards ( $\mathrm{CPH}$ ) model, incorporating factors that increased the probability of shorter survival: age at diagnosis, male gender, lower KPS, subtotal resection, and unmethylated MGMT status. The nomogram assesses survival probabilities (6-, 12-, and 24-mo) for patients with newly diagnosed glioblastoma and can be used to counsel patients regarding treatment decisions and optimizing duration of treatment and, like LGG counterpart, is available as free online software (16). 


\section{NOVEL APPROACHES TO SURVIVAL PREDICTION IN GLIOMA}

Novel approaches employ computational methods to generate survival prediction (Figure 1). The number of publications that analyze the relationship between clinical, pathological, histological and imaging factors, and survival in glioma have been increasing at a rapid pace (Figure 1). More than 50\% of publications involving computational approaches to survival prediction in glioma were generated in the last two years with more than 46 manuscripts dedicated to this subject (Figure 1A). Most of the manuscripts have focused on correlating imaging findings to survival prediction, followed closely by molecular characterization, genetics, and digital pathology (Figure 1B). Most data employed in novel computational approaches originated from smaller single-institution data sets with the remainder employing TCGA, Chinese Glioma Genome Atlas (CGGA) and Multimodal Brain Tumor Image Segmentation (BraTS) (Figure 1C). Only a small percentage of reports employed Surveillance, Epidemiology, and End Results (SEER) data (Figure $1 C$ ) presumably secondary to limitations currently inherent in nonspatial

A. Publication by year

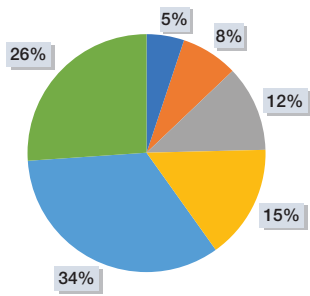

$$
\begin{aligned}
& \square 2008-2015 \\
& -2016 \\
& 2017 \\
& 2018 \\
& \square 2019 \\
& \square 2020
\end{aligned}
$$

C. Publication by cohort origin

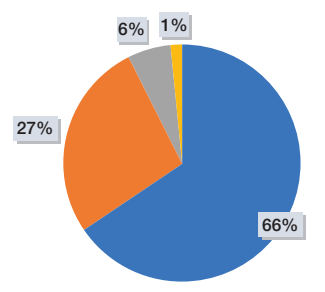

Institutional

- TCGA/CGGA

Dher registry

BraTs

- SEER

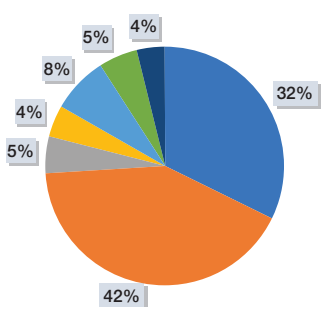

$$
\begin{aligned}
& \text { Genetics/Molecular } \\
& \text { Imaging } \\
& \text { Pathology } \\
& \text { Nonspatial data } \\
& \text { General/Multiple } \\
& \text { Radiation } \\
& \text { Segmentation }
\end{aligned}
$$

Figure 1. The landscape of computational approaches in the literature as related to glioma survival prediction. A. The $\%$ of publications related to glioma survival prediction by year of publication. B. The \% of publications related to glioma survival prediction grouped by the primary topic explored (as defined by the primary data type employed in the prediction analysis). C. The \% of publications related to glioma survival prediction grouped by the origin of the data employed to develop the approach. 
data sets including the lack of capture of molecular and pathological features, and management. Almost exclusively, the data currently employed in glioma survival prediction is retrospective in nature. Prospective data is being generated in a small cohort of ongoing clinical trials with robust histopathology, molecular, and imaging endpoints but may yet take some time to be incorporated in ongoing computational approaches (Tables 1 and 2). Computational approaches generally involve subcategories under the umbrella of artificial intelligence namely machine learning (ML) and deep learning (DL) (Figure 2).

\section{Machine learning approaches}

With increasing accessibility of electronic health records and large-scale registry data, ML has become an increasingly popular method to model survival. ML is a sub-field of AI where a computer algorithm automatically develops a model that transforms input data to output without using rules defined by humans. Classical ML methods require input data to have well defined sets of variables in the format of structured data (features). The process of extracting relevant structured variables from raw data to be used as ML input, known as feature engineering, often requires significant domain expertise and computational processing power, especially when it comes to input data such as images and natural language. DL is an emerging sub-field of ML where the DL algorithm can take raw data, such as images, as input and "learn" to define its own features needed for computing

\section{TABLE 1 Statistical analysis and machine learning terms employed in survival prediction}

\begin{tabular}{|c|c|}
\hline Term & Description \\
\hline COX/CPH model & $\begin{array}{l}\text { Cox's proportional hazards model: a regression model commonly investigating } \\
\text { the association between the survival time of patients and one or more } \\
\text { predictor variables (18). }\end{array}$ \\
\hline RPA & $\begin{array}{l}\text { Recursive Partitioning Analysis: a non-parametric statistical technique used to } \\
\text { create prognostic groups based on clinical features (1). }\end{array}$ \\
\hline C-index & $\begin{array}{l}\text { Concordance index: A performance metric for how well a model predicts the } \\
\text { ordering the patients' risk of death in comparison to ordering of patients' } \\
\text { recorded survival time (19). }\end{array}$ \\
\hline ROC & $\begin{array}{l}\text { Receiver operating characteristics curve: A probability curve of sensitivity } \\
\text { vs (1-specificity) when using different cutoff points in classifying binary } \\
\text { outcomes (19). }\end{array}$ \\
\hline AUC & $\begin{array}{l}\text { Area under the ROC curve: Area under the ROC that is used distinguishes the } \\
\text { discriminative potential of the algorithm (19). }\end{array}$ \\
\hline Model validation & $\begin{array}{l}\text { A ML trained model is evaluated with a test data set that is not used in training. } \\
\text { A popular validation method is } \mathrm{K} \text {-fold validation where the algorithm is } \\
\text { trained } \mathrm{K} \text { times with }(1 / \mathrm{K}) \text { of all data left out of training each time to be used } \\
\text { to evaluate model performance ( } 20 \text { ). }\end{array}$ \\
\hline
\end{tabular}




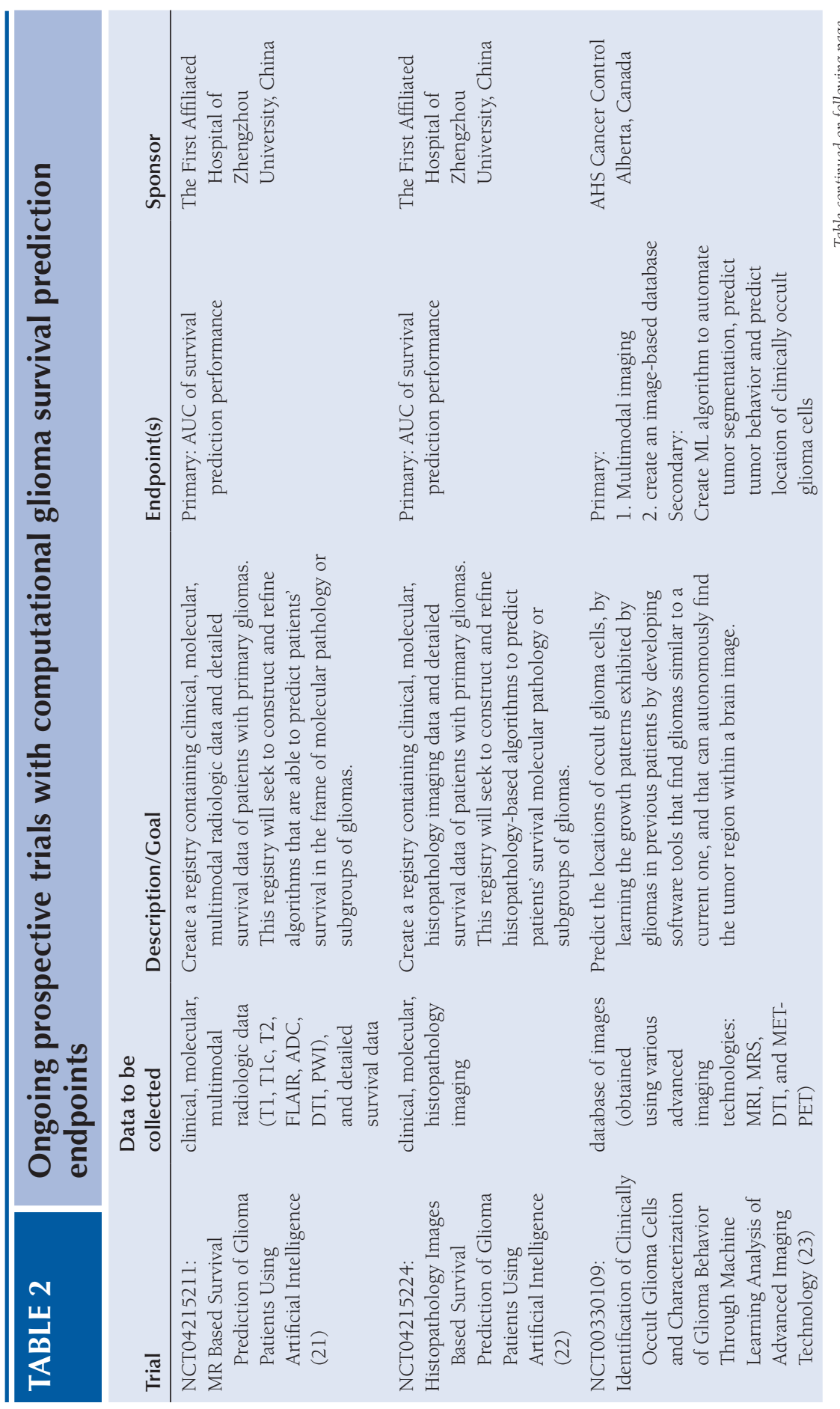




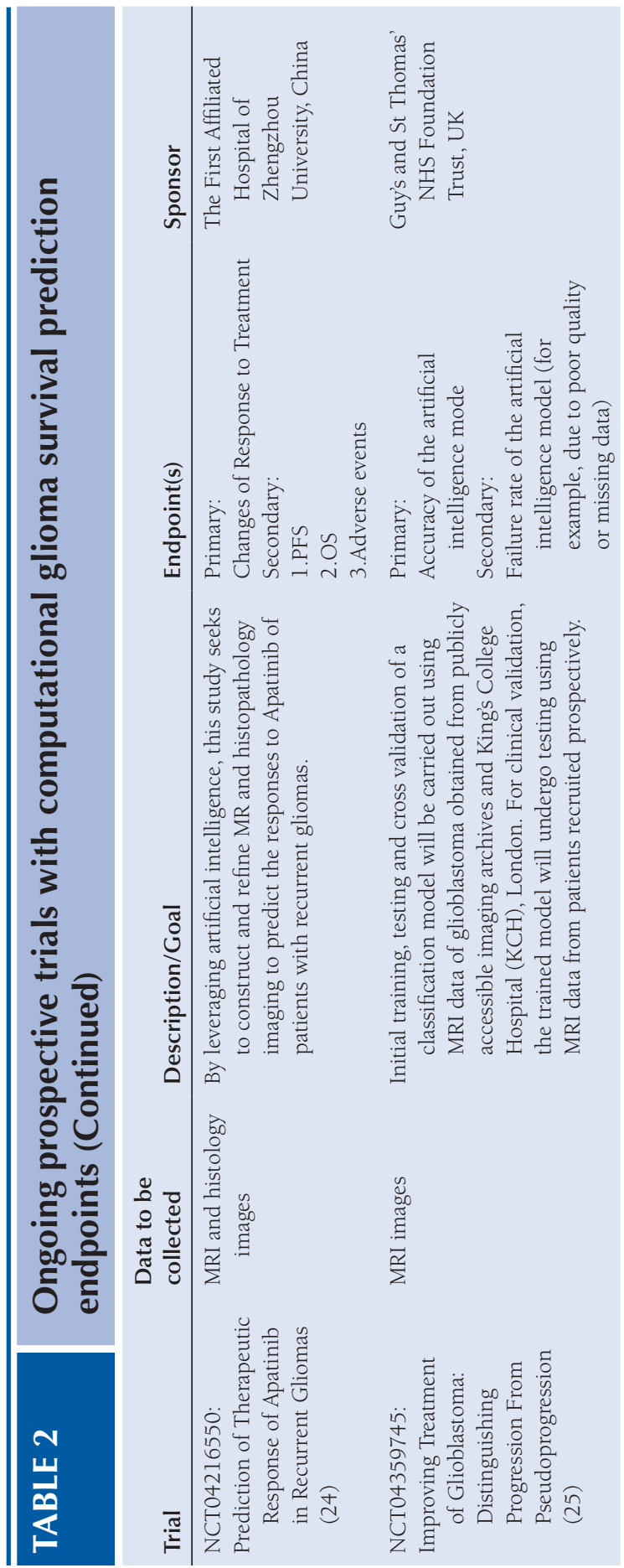




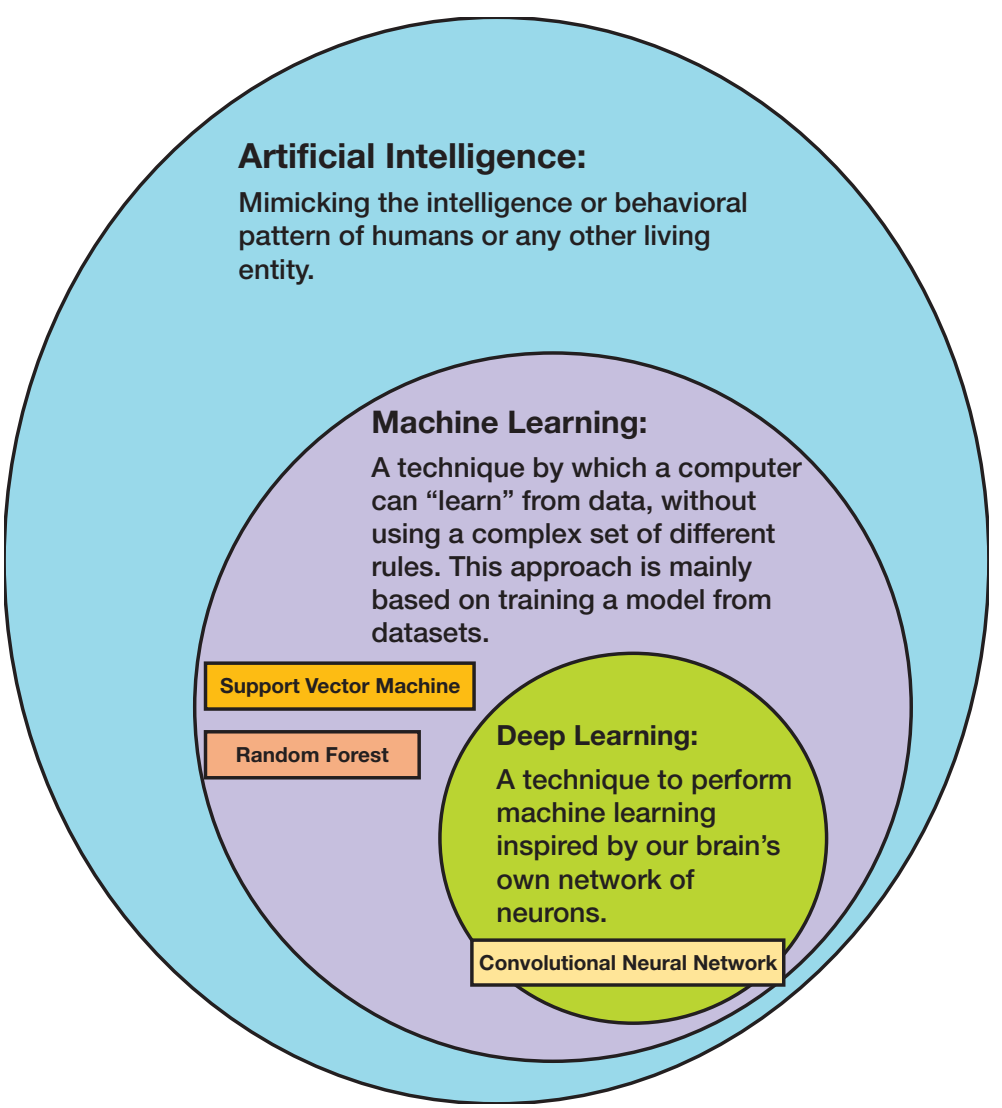

Figure 2. The relationship between Artificial intelligence (AI), Machine learning (ML) and Deep learning (DL) and models currently employed in survival analysis (26).

the outcome (27). ML and DL algorithms can be designed to capture the complexity of the patient profile by producing prognostic models that consider a large number of predictors including spatial and non-spatial data. Data extracted from large-scale registry and institutional data have been used for training ML and DL algorithms to improve the performance of survival outcome predictions. The data that have been used as training data include, but are not limited to, clinical characteristics, radiomics, histology, and molecular characteristics. Many studies have also shown different combinations of the use of imaging data (such as, Magnetic Resonance Imaging [MRI]) and Positron Emission Tomography [PET]) combined with clinical, histological, or genomic features (28-36). Some of the most popular ML algorithms used in survival prediction include support vector machine (SVM), random forest (RF) and a convolutional neural network (CNN) $(37,38)$. Their relationship with respect to AI, machine learning, and deep learning is illustrated in Figure 2. All three methods require a training step where example data, called training data, is used as an input into a learning 
algorithm which develops a model capable of mapping the input to an outcome as accurately as possible, and a prediction step where the trained model is used to predict outcomes given new data $(37,38)$. The individual approach inherent in each model is described below.

\section{Support vector machine}

SVM is a popular ML algorithm that has been used in classification and regression analysis in many fields of science. It can be successfully applied to analyze data with a large number of predictors and a limited sample size such as, thousands of radiomic features derived from imaging data, to predict survival outcome. The underlying principle of SVM is to optimize the separation of all observations into different classes (39). In the context of survival prediction, an SVM model can classify patients' survival time as long or short defined by an arbitrary survival time threshold used in model training. For example, Panesar et al. used the SVM algorithm to train models containing features including clinical and molecular characteristics, and WHO grade. A total of 76 patients were split into training and testing datasets in a 7:3 ratio randomly each time for 15 training cycles. The average performance of the resulting 15 models achieved a better binary classification performance (accuracy $=73.33 \%$ ) for 2-year survival than the benchmark statistical regression methods (accuracy $=69.27 \%)(34)$. Similarly, Sanghani et al. extracted 2200 radiomics features, including texture, volume and shape, from multi-channel MRI data of 163 patients and combined them with clinical features such as age and KPS. These features were used to train an SVM model which selected the top 150 most predictive features and used them to produce a model that classifies each patient's overall survival (OS) time into 2 classes (greater or less than 400 days) and 3 classes $(<10,10-15,>15$ months). The prediction accuracy was $98.7 \%$ for 2 -class, and $88.95 \%$ for 3 -class in cross-validation using internal data (29). Efforts have been made to adapt the SVM model for time-to-event analysis to predict survival time and improve its performance on right censored data by Khan et al. (40) and Van Belle et al. (41) by integrating regression constraints.

\section{Random forest}

Random forest $(\mathrm{RF})$ is a non-parametric ML algorithm that constructs multiple decision trees based on training features and uses the consensus or average of their output to get a more accurate prediction. Similar to SVM, RF algorithm can be used to model a large number of predictors with a limited number of observations $(18,42)$. For survival analysis, RF has been adapted by Ishwaran et al. to create a Random Survival Forest (RSF) capable of time-to-event analysis, taking into account right censored data (42). Audureau et al. trained two random forest models with different approaches, using clinical features including demographics, tumor location, KPS and treatment from 407 patients as a training set. The RF models performed slightly better than the gold standard statistical regression model (CPH model) when validated using external validation data (370 patients) with C-index of 70.14 and 70.37. Both RF models also identified KPS as the most important feature for predicting OS (43). Chang el al. trained a random 
forest model using features including volumetric, shape, texture, parametric, and histogram extracted from pretherapy (2293 features) and post-therapy (9811 features) multimodal MRI images of 84 patients to predict progression free survival (PFS) and OS. Long and short survival was defined as surviving more and less than the 50th percentile of PFS or OS in the training cohort respectively. An accuracy of 0.76 in classifying long and short survival was achieved in the validation cohort when using both pre- and post-therapy features, greatly exceeding the prediction accuracy of models trained only using pre-therapy features which was 0.57 and 0.54 , respectively (35).

\section{Convolutional neural network}

Convolutional neural network (CNN) is a deep learning technique widely used in image analysis. A CNN can learn to classify or identify objects in images by automatically learning to extract features from them instead of using human defined features to interpret the images. For example, Mobadersany et al. developed a survival convolutional neural network (GSCNN) that integrated raw histological images with genomic biomarkers (IDH mutation status and 1q/19q codeletion) from 769 patients to produce a survival prediction framework with prognostic accuracy $(\mathrm{C}$-index $=0.801$ ) surpassing the $\mathrm{WHO}$ paradigm based on genomic classification or histological grading (36) when tested using internal data. Nie et al. trained a CNN to classify segments of whole brain images from 68 patients into long and short survival time (threshold at 650 days) with over $80 \%$ accuracy. The "deep features" learned by CNN, along with clinical features of patients, were then used as input for an SVM model which achieved an accuracy of over 90.66\% in classifying long and short survival when validated on independent dataset (32). These studies highlighted the ability of CNN to automatically extract features predictive of survival from raw images. The generalizability and transferability of these high-level image features still require testing using data from various external institutions.

\section{Evaluating machine learning approaches}

Concordance index (C-index) is the most commonly used metric for evaluating survival predictions. It measures the accuracy of the model's predictions of the ordering of patients' risk of death (equivalent to survival time ranking) in comparison to ordering of patients' recorded survival time. It is a value ranging from 0.5 (indicating random ordering by model) to 1 (indicating perfect concordant ordering by model) and can also be estimated for right censored data $(19,44)$ (Table 1). Most ML and DL studies have achieved a C-index above 0.7 (31, 36, 45-47). For discrete survival classification using a survival time threshold, the overall classification accuracy is often calculated.

\section{Radiomics and other computational approaches}

Radiomics-type approaches are becoming increasingly common. They share an approach to inferring tumor grading, molecular features and/or tumor behavior following treatment in conjunction with tumor imaging and linking this to 
outcome prediction including survival (46-48). Broadly, radiomics is an emerging field that involves extraction and quantification of features from medical images (48). The data can then be analyzed using computational analysis and models to identify predictive image biomarkers that characterize tumor behavior. Jan et al. carried out an extensive literature review of radiomics-based analyses, with a particular focus on computational modeling, machine learning, and fractalbased analysis aimed at optimizing differential diagnosis and predicting clinical outcomes. Han et al. combined a deep learning and radiomics model to predict OS in a cohort of 50 patients from their institution and 128 patients from TCGA with high-grade glioma. They calculated 348 radiomics features and 8192 deep features generated by a pretrained convolutional neural network and then applied feature selection and Elastic Net-Cox modeling to differentiate patients into longand short-term survivors (46). Similarly, Lao et al. employed deep features to generate radiomic signatures for prediction of OS in a data set of 75 patients and an independent validation data set of 37 patients with glioblastoma. They extracted a total of 1403 handcrafted features and 98304 deep features from MRI and generated a radiomics nomogram combining the signature and clinical risk factors such as age and KPS. The proposed signature achieved better performance for prediction of survival and significant stratification of patients into prognostically distinct groups with a C-index of 0.739 , demonstrating that a prognostic imaging signature exists and patient stratification for glioblastoma was possible (47). While most studies employ MRI, functional imaging in the form of ${ }^{18} \mathrm{~F}$-fluorodeoxyglucose positron emission tomography $\left({ }^{18} \mathrm{~F}-\mathrm{FDG} \mathrm{PET}\right.$ ) has also been employed in radiomics analyses $(30,49)$. Li et al. employed the images of 127 patients to develop a model to analyze features reflecting glioma metabolism for predicting IDH genotype and prognosis and generated a radiomic signature significantly associated with IDH genotype (49).

\section{Imaging and tumor grading-based survival prediction}

By far the most common theme in computational glioma survival prediction involving imaging relates to glioma tumor grading. Numerous publications have employed tumor grading from imaging features as a starting point to help develop prognostic biomarkers $(21,50-60)$. Some publications have focused strictly on glioma grading $(31,50,58)$ while others explored more specific molecular subtypes (54) and IDH mutation status $(51-53,57,58)$. Juan-Albarracin et al. developed ONCOhabitats (https://www.oncohabitats.upv.es [accessed on 10 December 2020]): an online open access system for glioblastoma analysis based on MRI data, including malignant tissue segmentation and vascular heterogeneity assessment of the tumor while implementing a deep patch-wise 3D CNN with residual connections. This allows open-access services for glioblastoma heterogeneity assessment and medical image analysis with a computational capacity of 300 cases per day (50). Tan et al. analyzed the clinical data, genetic features and MRI images of 147 high-grade glioma (112 patients as training cohort, 35 as independent test cohort) to develop a radiomic signature to predict OS for HGG, and constructed a nomogram by combining selected radiomic, genetic and clinical risk factors. The radiomics features were extracted from the tumor area and the peritumoral edema area on CE-TIWI (contrast-enhanced T1-weighted imaging) 
and T2FLAIR (T2 fluid-attenuated inversion recovery) images, and the risk factors and OS were explored by Kaplan-Meier survival analysis by stratifying patients into low- and high-risk groups (C-Index 0.707 and 0.711 in training and test cohorts respectively) (31). By contrast, using CNN Zhuge et al. created two fully automated glioma grading methods on conventional MRI images that were then evaluated on The Cancer Imaging Archive (TCIA) LGG data, and the BraTS Benchmark 2018 training datasets (55). In yet another approach, Liao et al. examined multi-dimensional MRI features extracted from segmented lesions of T2-FLAIR MRI data of 137 glioblastoma patients together with RNA sequencing in groups of glioblastoma patients identifying radiomic parameters including intensity, shape and textural features that were incorporated into seven classes to divide the patient cohort into two groups depending on their survival time, concluding that MRI features are predictive of survival outcomes and image features are highly associated with selective metagenes (59).

With respect to molecular classification in terms of IDH mutation status, Suchorska et al. stratified 301 patients with WHO grade II $(n=181)$ or grade III glioma $(\mathrm{n}=120)$ according to their molecular profile and reviewed pre-operative MRI and volumetric analyses of contrast-enhanced and T2 volumes, showing that contrast enhancement on initial MRI is a prognostic factor for survival with dependence on volume distinguishing IDH-mutated from IDH-wild type tumors (51). Similarly, with an eye towards predicting IDH mutation pre-operatively, several publications have employed MRI and ML $(57,58,61)$. Zhang et al. employed preoperative MRIs of 120 HGG patients with confirmed IDH genotype, to extract 2970 imaging features from pre- and post-contrast T1-weighted, T2-weighted, and apparent diffusion coefficient (ADC) maps. Using RF, the preoperative MRI features with the highest predictive value for IDH genotype were patient age, parametric intensity, texture, and shape (57). Similar analyses using preoperative prediction of IDH status were carried out by Tan et al. in 105 astrocytoma (Grades II-IV) (58).

\section{Imaging and response to treatment}

The use of imaging to assess response to treatment in glioma is of tremendous importance for both patient management and outcome assessment. With respect to computational approaches on analyzing survival outcomes, research to date has focused on two main aspects: (i) distinguishing progression from pseudo-progression $(62,63)$; and (ii) connecting systemic therapy or radiation therapy (RT) to imaging changes (64). Artzi et al. utilized data generated using conventional, dynamic contrast enhanced (DCE)-MRI and magnetic resonance spectroscopy from 20 patients to extract the enhanced lesion area using independent component analysis and choline/creatine values and compared treatment-related changes with normal-appearing white matter. They identified a progressive disease component within the lesion, concluding that the results may have clinical importance for preoperative planning, guidance for targeting biopsy and early prediction of radiological outcomes in patients with HGG (62). Kebir et al. carried out a similar analysis using 14 patients with HGG suspected of progression but used FET-PET imaging to identify 3 clusters based on 10 predominantly textural FET-PET features. Similar studies were also carried out by Chan et al. and Petrova et al. $(64,65)$. 
Correlating response to RT with images using $\mathrm{ML}$ and $\mathrm{DL}$ is challenging. Mizutani et al. employed scans of 35 patients with malignant glioma, identifying 12 clinical features and 192 dose-volume histogram (DVH) features and used SVM to predict OS times after RT. They found that prediction accuracy was significantly improved with the combined use of clinical and DVH features compared with the separate use of each feature (66). Qiu et al. compared RSF and traditional $\mathrm{CPH}$ to predict tumor progression after particle beam radiotherapy in 82 HGG patients and found that CPH demonstrated a better performance in terms of integrated C-index as compared to the RSF model (18).

The question as to the most optimal computational approach to be employed in glioma survival prediction pertaining to imaging data is as yet unresolved (32, 55, 67-69). Zacharaki et al. (28) suggested that prediction models based on data-mining algorithms can provide a more accurate information about prognosis of malignant gliomas than histopathologic classification alone. Since then, more studies have concluded that the combination of clinical and multi feature imaging is crucial to obtain accurate model prediction $(39,45,70)$. Some studies also suggest that DL approaches may be superior to $\operatorname{ML}(32,67,68)$. Mirroring traditional RPA approaches to survival prediction, some publications aim to classify gliomas by survival time using DL techniques (71) and more novel approaches. For example, Smedley et al. describe a neural network-based approach that takes highdimensional gene expression data as input and performs non-linear mapping to an imaging trait, identifying imaging traits with specific transcription patterns, such as edema and genes related to cellular invasion (72). Mostly, small institutional cohorts are being employed as opposed to large registries such as TCGA $(73,74)$. Studies suggests that imaging-based glioma survival prediction carries greater potential as compared to traditional approaches to what is currently radiographically being identified as gross tumor on scans $(67,74)$. Brain tumor segmentation also remains an active area of research that has significant implications for computational approaches (75-77). Currently, most studies are based on very small institutional cohorts (Figure 1 ) and the validation of models is heterogenous in the literature, although with ongoing research this is likely to change rapidly in the coming years.

\section{Molecular and genetic characterization of glioma, digital pathology, and survival prediction}

Several molecular markers such as IDH1/2, 1p19q co-deletion, TERT and MGMT promoter methylation, G-CIMP methylation, EGFR alternations, BRAF V600E mutations and histone mutations have been found to have prognostic significance in glioma (78). Statistical methods can be applied to stratify prognosis based on molecular characteristics. Bell et al. created a new RPA model (NRG-GBM-RPA) that creates distinct prognostic groups based on age, MGMT protein and c-Met protein levels. The new model resulted in improved survival stratification in patients with glioblastoma treated with RT and temozolomide in comparison with current RPA classifications based on age, KPS, resection status and neurofunction status (79).

In the 2019 "Contributions from the 2018 Literature on Bioinformatics and Translational Informatics" (80), the two primary trends identified were: 
(i) the adoption of artificial intelligence and DL methods in medical informatics; and (ii) the implementation of a pan-cancer approach and integration of multiomics data for more insightful analyses (80). In glioma, RNA based biomarkers are evolving and traditional statistical models have been employed to create prognostic groups $(81,82)$, while ongoing efforts using computational approaches that involve ML and DL are advancing for somatic copy number variations (83) and gene expression microarrays (84). To date, both $1 \mathrm{pl9q}(85)$ and MGMT $(86,87)$ are important molecular features undergoing active inclusion into computational approaches to glioma survival prediction. Transcriptomic analyses using TCGA glioma expression datasets are being advanced to identify novel tumor subcategories using ML $(88,89)$. Panesar et al. applied 3 ML techniques (CNN, RF, SVM), and classical logistic regression to the molecular characteristics of a dataset consisting of 76 patients with glioma of all grades achieving reasonable performance compared with similar studies in the literature, but noted that, similar to other studies, traditional statistical methods were of similar benefit (34). This illustrates that more research is needed particularly with larger data sets and validation.

Digital pathology is emerging as an increasingly important facet of the approach to glioma pathology and classification and has been employed in both ML and DL approaches to integrate information from both histology images and genomic biomarkers to predict time-to-event outcomes (36). It has been utilized for wholeslide imaging of histologic sections to extract quantitative features (90). Powell et al. used hematoxylin- and eosin-stained slides from TCGA to create a machine learned dictionary of "image-derived visual words" associated with survival outcomes while connecting image-derived phenotypic characteristics with molecular signaling activity and the behavior of low-grade glioma (91).

\section{CONCLUSION}

Retrospective spatial and nonspatial data from patients with glioma is increasingly available and prospective data is being generated to provide an avenue for novel approaches to survival prediction. While many computational approaches show promising performance in terms of survival prediction accuracy, most ML prognostic models are trained using data from single-institutions and have not been validated using external cohorts. To facilitate the implementation of ML prognostic models into clinical practice, prospective validation of these models on large scale heterogenous cohorts from multiple centers would be required (92). Digital pathology is an exciting avenue being advanced to explore survival prediction in glioma. Most importantly, the neuro-oncology field needs to familiarize itself with computational approaches and quality metrics for the assessment of such approaches to ensure robust conclusions that drive improvement in patient outcomes in the clinic. There is a growing need to foster reliable clinician/ ML innovation to support the generation of robust data sets in large scale registries such as TCGA, SEER and BraTS. Efforts towards developing consensus and clinical oversight in the methods for data acquisition and coding across different institutions could facilitate external and prospective validation of survival prediction models. 
Conflict of Interest: The authors declare no potential conflict of interest with respect to research, authorship and/or publication of this chapter.

Copyright and permission statement: The authors confirm that the materials included in this chapter do not violate copyright laws. Where relevant, appropriate permissions have been obtained from the original copyright holder(s), and all original sources have been appropriately acknowledged or referenced.

\section{REFERENCES}

1. Wee CW, Kim IH, Park CK, Kim JW, Dho YS, Ohka F, et al. Validation of a novel molecular RPA classification in glioblastoma (GBM-molRPA) treated with chemoradiation: A multi-institutional collaborative study. Radiother Oncol. 2018;129(2):347-51. https://doi.org/10.1016/j.radonc.2018.09.001

2. Bell EH, Pugh SL, McElroy JP, Gilbert MR, Mehta M, Klimowicz AC, et al. Molecular-Based Recursive Partitioning Analysis Model for Glioblastoma in the Temozolomide Era: A Correlative Analysis Based on NRG Oncology RTOG 0525. JAMA Oncol. 2017;3(6):784-92.

3. Curran WJ Jr, Scott CB, Horton J, Nelson JS, Weinstein AS, Fischbach AJ et al. Recursive partitioning analysis of prognostic factors in three Radiation Therapy Oncology Group malignant glioma trials. J Natl Cancer Inst. 1993; 85(9):704-10. https://doi.org/10.1093/jnci/85.9.704

4. Li J, Wang M, Won M, Coughlin C, Curran WJ, Mehta MP, et al. Validation and simplification of the Radiation Therapy Oncology Group recursive partitioning analysis classification for glioblastoma. Int J Radiat Oncol Biol Phys. 2011; 81(3):623-30. https://doi.org/10.1016/j.ijrobp.2010.06.012

5. Zanello M, Roux A, Ursu R, Peeters S, Bauchet L, Noel G, et al; On the Behalf of the Club de NeuroOncologie of the Société Française de Neurochirurgie. Recurrent glioblastomas in the elderly after maximal first-line treatment: does preserved overall condition warrant a maximal second-line treatment? J Neurooncol. 2017;135(2):285-97. https://doi.org/10.1007/s11060-017-2573-y

6. Ius T, Somma T, Altieri R, Angileri FF, Barbagallo GM, Cappabianca P, et al. Is age an additional factor in the treatment of elderly patients with glioblastoma? A new stratification model: an Italian Multicenter Study. Neurosurg Focus. 2020;49(4):E13. https://doi.org/10.3171/2020.7.FOCUS20420

7. Yesil Cinkir H, Colakoglu Er H. Is temporal muscle thickness a survival predictor in newly diagnosed glioblastoma multiforme? Asia Pac J Clin Oncol. 2020;16(5):e223-e227. https://doi.org/10.1111/ ajco. 13369

8. Lütgendorf-Caucig C, Freyschlag C, Masel EK, Marosi C. Guiding Treatment Choices for Elderly Patients with Glioblastoma by a Comprehensive Geriatric Assessment. Curr Oncol Rep. 2020;22(9):93. https://doi.org/10.1007/s11912-020-00951-6

9. Lorimer CF, Walsh G, MacKinnon M, Corbett A, Bedborough K, Greenwood K, et al. Geriatric assessment of glioblastoma patients is feasible and may provide useful prognostic information. Neurooncol Pract. 2020;7(2):176-84. https://doi.org/10.1093/nop/npz040

10. Straube C, Kessel KA, Antoni S, Gempt J, Meyer B, Schlegel J, et al. A balanced score to predict survival of elderly patients newly diagnosed with glioblastoma. Radiat Oncol. 2020;15(1):97. https://doi. org/10.1186/s13014-020-01549-9

11. Gittleman H, Sloan AE, Barnholtz-Sloan JS. An independently validated survival nomogram for lowergrade glioma. Neuro Oncol. 2020;22(5):665-74. https://doi.org/10.1093/neuonc/nozl91

12. Lombardi G, Barresi V, Castellano A, Tabouret E, Pasqualetti F, Salvalaggio A, et al. Clinical Management of Diffuse Low-Grade Gliomas. Cancers (Basel). 2020;12(10):3008. https://doi.org/ $10.3390 /$ cancers 12103008

13. Rudà R, Touat $\mathrm{M}$, Soffietti R. Is chemotherapy alone an option as initial treatment for low-grade oligodendrogliomas? Curr Opin Neurol. 2020;33(6):707-15. https://doi.org/10.1097/WCO. 0000000000000866 
14. Ryckman JM, Appiah AK, Lyden E, Verma V, Zhang C. Concurrent Versus Sequential Chemoradiation for Low-grade Gliomas Meeting RTOG 9802 Criteria. Am J Clin Oncol. 2019;42(4):391-98. https:// doi.org/10.1097/COC.0000000000000519

15. Topkan E, Selek U, Ozdemir Y, Yildirim BA, Guler OC, Ciner F, et al. Prognostic value of the Glasgow Prognostic Score for glioblastoma multiforme patients treated with radiotherapy and temozolomide. J Neurooncol. 2018;139(2):411-419. https://doi.org/10.1007/s11060-018-2879-4

16. Gittleman H, Lim D, Kattan MW, Chakravarti A, Gilbert MR, Lassman AB, et al. An independently validated nomogram for individualized estimation of survival among patients with newly diagnosed glioblastoma: NRG Oncology RTOG 0525 and 0825. Neuro Oncol. 2017;19(5):669-77. https://doi. org/10.1093/neuonc/now208

17. Villano JL, Letarte N, Bressler LR. Going past the data for temozolomide. Cancer Chemother Pharmacol. 2012;69(4):1113-5. https://doi.org/10.1007/s00280-011-1796-4

18. Qiu X, Gao J, Yang J, Hu J, Hu W, Kong L, et al. A Comparison Study of Machine Learning (Random Survival Forest) and Classic Statistic (Cox Proportional Hazards) for Predicting Progression in HighGrade Glioma after Proton and Carbon Ion Radiotherapy. Front Oncol. 2020;10:551420 https://doi. org/10.3389/fonc. 2020.551420

19. Chen H, Kodell RL, Cheng KF, Chen JJ. Assessment of performance of survival prediction models for cancer prognosis. BMC Med Res Methodol. 2012;12(1):102. https://doi.org/10.1186/1471-2288-12-102

20. Bengio Y, Grandvalet Y. No unbiased estimator of the variance of k-fold cross-validation. J Mach Learn Res. 2004;1089-1105.

21. National Library of Medicine (U.S.). (2017, January - ). MR Based Survival Prediction of Glioma Patients Using Artificial Intelligence. Identifier NCT04215211. https://clinicaltrials.gov/ct2/show/ NCT04215211 [Accessed on 15 Mar 2021]

22. National Library of Medicine (U.S.). (2017, January - ). Histopathology Images Based Survival Prediction of Glioma Patients Using Artificial Intelligence. Identifier NCT04215224. https://clinicaltrials.gov/ct2/show/NCT04215224 [Accessed on 15 Mar 2021]

23. National Library of Medicine (U.S.). (2017, December - ). Identification of Clinically Occult Glioma Cells and Characterization of Glioma Behavior Through Machine Learning Analysis of Advanced Imaging Technology. Identifier NCT00330109. https://clinicaltrials.gov/ct2/show/NCT00330109 [Accessed on 15 Mar 2021]

24. National Library of Medicine (U.S.). (2018, January - ). Prediction of Therapeutic Response of Apatinib in Recurrent Gliomas. Identifier NCT04216550. https://clinicaltrials.gov/ct2/show/NCT04216550 [Accessed on 15 Mar 2021]

25. National Library of Medicine (U.S.). (2019, March - ). Image-derived Prediction of Response to Chemo-radiation in Glioblastoma (IDEPREG). Identifier NCT02329795. https://clinicaltrials.gov/ct2/ show/NCT02329795 [Accessed on 15 Mar 2021]

26. Original file: Avimanyu786SVG version: Tukijaaliwa, CC BY-SA 4.0, https://creativecommons.org/ licenses/by-sa/4.0, via Wikimedia Common. [Accessed on 15 Mar 2021]

27. Esteva A, Robicquet A, Ramsundar B, Kuleshov V, DePristo M, Chou K, et al. A guide to deep learning in healthcare. Nat Med. 2019;25(1):24-9. https://doi.org/10.1038/s41591-018-0316-z

28. Zacharaki EI, Morita N, Bhatt P, O'Rourke DM, Melhem ER, Davatzikos C. Survival analysis of patients with high-grade gliomas based on data mining of imaging variables. Am J Neuroradiol. 2012;33(6):1065-71. https://doi.org/10.3174/ajnr.A2939

29. Sanghani P, Ang BT, King NKK, Ren H. Overall survival prediction in glioblastoma multiforme patients from volumetric, shape and texture features using machine learning. Surg Oncol. 2018;27(4):709-14. https://doi.org/10.1016/j.suronc.2018.09.002

30. Papp L, Pötsch N, Grahovac M, Schmidbauer V, Woehrer A, Preusser M, et al. Glioma Survival Prediction with Combined Analysis of In Vivo 11 C-MET PET Features, Ex Vivo Features, and Patient Features by Supervised Machine Learning. J Nucl Med. 2018; 59(6):892-99. https://doi.org/10.2967/ jnumed.117.202267

31. Tan Y, Mu W, Wang X, Yang G, Gillies RJ, Zhang H. Improving survival prediction of high-grade glioma via machine learning techniques based on MRI radiomic, genetic and clinical risk factors. Eur J Radiol. 2019;120:108609. https://doi.org/10.1016/j.ejrad.2019.07.010 
32. Nie D, Lu J, Zhang H, Adeli E, Wang J, Yu Z, et al. Multi-Channel 3D Deep Feature Learning for Survival Time Prediction of Brain Tumor Patients Using Multi-Modal Neuroimages. Sci Rep. 2019;9(1):1103. https://doi.org/10.1038/s41598-018-37387-9

33. Tang Z, Xu Y, Jin L, Aibaidula A, Lu J, Jiao Z, et al. Deep Learning of Imaging Phenotype and Genotype for Predicting Overall Survival Time of Glioblastoma Patients. IEEE Trans Med Imaging. 2020;39(6):2100-09. https://doi.org/10.1109/TMI.2020.2964310

34. Panesar SS, D'Souza RN, Yeh F, Fernandez-Miranda JC. Machine Learning Versus Logistic Regression Methods for 2-Year Mortality Prognostication in a Small, Heterogeneous Glioma Database. World Neurosurg X. 2019;2:100012. https://doi.org/10.1016/j.wnsx.2019.100012

35. Chang K, Zhang B, Guo X, Zong M, Rahman R, Sanchez D, et al. Multimodal imaging patterns predict survival in recurrent glioblastoma patients treated with bevacizumab. Neuro-oncology. 2016;18(12):1680-87. https://doi.org/10.1093/neuonc/now086

36. Mobadersany P, Yousefi S, Amgad M, Gutman DA, Barnholtz-Sloan JS, Velázquez Vega JE, et al. Predicting cancer outcomes from histology and genomics using convolutional networks. Proc Natl Acad Sci U S A. 2018;115(13):E2970-E2979. https://doi.org/10.1073/pnas.1717139115

37. Sidey-Gibbons JAM, Sidey-Gibbons CJ. Machine learning in medicine: a practical introduction. BMC Med Res Methodol. 2019;19(1):64. https://doi.org/10.1186/s12874-019-0681-4

38. Sotoudeh H, Shafaat O, Bernstock JD, Brooks MD, Elsayed GA, Chen JA, et al. Artificial Intelligence in the Management of Glioma: Era of Personalized Medicine. Front Oncol. 2019; 9:768. https://doi. org/10.3389/fonc. 2019.00768

39. Sanz H, Reverter F, Valim C. Enhancing SVM for survival data using local invariances and weighting. BMC Bioinformatics. 2020;21(1):193. https://doi.org/10.1186/s12859-020-03558-7

40. Khan FM, Bayer Zubek V. "Support vector regression for censored data (SVRc): a novel tool for survival analysis." 2008 Eighth IEEE International Conference on Data Mining. IEEE, 2008. https://doi. org/10.1109/ICDM.2008.50

41. Van Belle V, Pelckmans K, Van Huffel S, Suykens JA. "Support vector methods for survival analysis: a comparison between ranking and regression approaches." Artif Intell Med. 2011; 53(2):107-18. https://doi.org/10.1016/j.artmed.2011.06.006

42. Ishwaran H, Kogalur UB, Blackstone EH, Lauer MS. "Random survival forests." Ann Appl Stat. 2008; 841-860. https://doi.org/10.1214/08-AOAS169

43. Audureau E, Chivet A, Ursu R, Corns R, Metellus P, Noel G, et al. Prognostic factors for survival in adult patients with recurrent glioblastoma: a decision-tree-based model. J Neurooncol 2018;136(3): 565-76. https://doi.org/10.1007/s11060-017-2685-4

44. Uno H, Cai T, Pencina MJ, D'Agostino RB, Wei LJ. On the C-statistics for evaluating overall adequacy of risk prediction procedures with censored survival data. Stat Med. 2011;30(10):1105. https://doi. org/10.1002/sim.4154

45. Peeken JC, Goldberg T, Pyka T, Bernhofer M, Wiestler B, Kessel KA, et al. Combining multimodal imaging and treatment features improves machine learning-based prognostic assessment in patients with glioblastoma multiforme. Cancer Med. 2019;8(1):128-36. https://doi.org/10.1002/ cam4.1908

46. Han W, Qin L, Bay C, Chen X, Yu KH, Miskin N, et al. Deep Transfer Learning and Radiomics Feature Prediction of Survival of Patients with High-Grade Gliomas. Am J Neuroradiol. 2020;41(1):40-8. https://doi.org/10.3174/ajnr.A6365

47. Lao J, Chen Y, Li ZC, Li Q, Zhang J, Liu J, et al. A Deep Learning-Based Radiomics Model for Prediction of Survival in Glioblastoma Multiforme. Sci Rep. 2017;7(1):10353. https://doi.org/10.1038/ s41598-017-10649-8

48. Jang K, Russo C, Di leva A. Radiomics in gliomas: clinical implications of computational modeling and fractal-based analysis. Neuroradiol. 2020;62(7):771-90. https://doi.org/10.1007/s00234-020-02403-1

49. Li L, Mu W, Wang Y, Liu Z, Liu Z, Wang Y, et al. A Non-invasive Radiomic Method Using 18F-FDG PET Predicts Isocitrate Dehydrogenase Genotype and Prognosis in Patients With Glioma. Front Oncol. 2019;9:1183. https://doi.org/10.3389/fonc.2019.01183

50. Juan-Albarracín J, Fuster-Garcia E, García-Ferrando GA, García-Gómez JM. ONCOhabitats: A system for glioblastoma heterogeneity assessment through MRI. Int J Med Inform. 2019;128:53-61. https:// doi.org/10.1016/j.ijmedinf.2019.05.002 
51. Suchorska B, Schüller U, Biczok A, Lenski M, Albert NL, Giese A, et al. Contrast enhancement is a prognostic factor in IDH1/2 mutant, but not in wild-type WHO grade II/III glioma as confirmed by machine learning. Eur J Cancer. 2019;107:15-27 https://doi.org/10.1016/j.ejca.2018.10.019

52. Chang K, Bai HX, Zhou H, Su C, Bi WL, Agbodza E, et al. Residual Convolutional Neural Network for the Determination of IDH Status in Low- and High-Grade Gliomas from MR Imaging. Clin Cancer Res. 2018;24(5):1073-81. https://doi.org/10.1158/1078-0432.CCR-17-2236

53. Akkus Z, Ali I, Sedlář J, Agrawal JP, Parney IF, Giannini C, et al. Predicting Deletion of Chromosomal Arms 1p/19q in Low-Grade Gliomas from MR Images Using Machine Intelligence. J Digit Imaging. 2017;30(4):469-476. https://doi.org/10.1007/s10278-017-9984-3

54. Macyszyn L, Akbari H, Pisapia JM, Da X, Attiah M, Pigrish V, et al. Imaging patterns predict patient survival and molecular subtype in glioblastoma via machine learning techniques. Neuro Oncol. 2016;18(3):417-25. https://doi.org/10.1093/neuonc/nov127

55. Zhuge Y, Ning H, Mathen P, Cheng JY, Krauze AV, Camphausen K, et al. Automated glioma grading on conventional MRI images using deep convolutional neural networks. Med Phys. 2020;47(7):3044-53. https://doi.org/10.1002/mp.14168

56. Choi YS, Ahn SS, Chang JH, Kang SG, Kim EH, Kim SH, et al. Machine learning and radiomic phenotyping of lower grade gliomas: improving survival prediction. Eur Radiol. 2020;30(7):3834-42. https://doi.org/10.1007/s00330-020-06737-5

57. Zhang B, Chang K, Ramkissoon S, Tanguturi S, Bi WL, Reardon DA, et al. Multimodal MRI features predict isocitrate dehydrogenase genotype in high-grade gliomas. Neuro Oncol. 2017;19(1):109-17. https://doi.org/10.1093/neuonc/nowl21

58. Tan Y, Zhang ST, Wei JW, Dong D, Wang XC, Yang GQ, et al. A radiomics nomogram may improve the prediction of IDH genotype for astrocytoma before surgery. Eur Radiol. 2019;29(7):3325-37. https:// doi.org/10.1007/s00330-019-06056-4

59. Liao X, Cai B, Tian B, Luo Y, Song W, Li Y. Machine-learning based radiogenomics analysis of MRI features and metagenes in glioblastoma multiforme patients with different survival time. J Cell Mol Med. 2019;23(6):4375-85. https://doi.org/10.1111/jcmm.14328

60. Liu X, Li Y, Sun Z, Li S, Wang K, Fan X, et al. Molecular profiles of tumor contrast enhancement: A radiogenomic analysis in anaplastic gliomas. Cancer Med. 2018;7(9):4273-83. https://doi. org/10.1002/cam4.1672

61. Kesler SR, Harrison RA, Petersen ML, Rao V, Dyson H, Alfaro-Munoz K, et al. Pre-surgical connectome features predict IDH status in diffuse gliomas. Oncotarget. 2019;10(60):6484-93. https://doi. org/10.18632/oncotarget.27301

62. Artzi M, Liberman G, Nadav G, Blumenthal DT, Bokstein F, Aizenstein O, et al. Differentiation between treatment-related changes and progressive disease in patients with high grade brain tumors using support vector machine classification based on DCE MRI. J Neurooncol. 2016;127(3):515-24. https://doi.org/10.1007/s11060-016-2055-7

63. Kebir S, Khurshid Z, Gaertner FC, Essler M, Hattingen E, Fimmers R, et al. Unsupervised consensus cluster analysis of [18F]-fluoroethyl-L-tyrosine positron emission tomography identified textural features for the diagnosis of pseudoprogression in high-grade glioma. Oncotarget. 2017;8(5):82948304. https://doi.org/10.18632/oncotarget.14166

64. Chang Y, Lafata K, Sun W, Wang C, Chang Z, Kirkpatrick JP, et al. An investigation of machine learning methods in delta-radiomics feature analysis. PLoS One. 2019;14(12):e0226348. https://doi. org/10.1371/journal.pone.0226348

65. Petrova L, Korfiatis P, Petr O, LaChance DH, Parney I, Buckner JC, et al. Cerebral blood volume and apparent diffusion coefficient - Valuable predictors of non-response to bevacizumab treatment in patients with recurrent glioblastoma. J Neurol Sci. 2019;405:116433. https://doi.org/10.1016/j. jns.2019.116433

66. Mizutani T, Magome T, Igaki H, Haga A, Nawa K, Sekiya N, et al. Optimization of treatment strategy by using a machine learning model to predict survival time of patients with malignant glioma after radiotherapy. J Radiat Res. 2019;60(6):818-824. https://doi.org/10.1093/jrr/rrz066

67. Molina-García D, Vera-Ramírez L, Pérez-Beteta J, Arana E, Pérez-García VM. Prognostic models based on imaging findings in glioblastoma: Human versus Machine. Sci Rep. 2019;9(1):5982. https://doi. org/10.1038/s41598-019-42326-3 
68. Hao J, Kim Y, Kim TK, Kang M. PASNet: pathway-associated sparse deep neural network for prognosis prediction from high-throughput data. BMC Bioinformatics. 2018;19(1):510. https://doi. org/10.1186/s12859-018-2500-z

69. Zhang Y, Li A, Peng C, Wang M. Improve Glioblastoma Multiforme Prognosis Prediction by Using Feature Selection and Multiple Kernel Learning. IEEE/ACM Trans Comput Biol Bioinform. 2016;13(5):825-35. https://doi.org/10.1109/TCBB.2016.2551745

70. Liu L, Zhang H, Wu J, Yu Z, Chen X, Rekik I, et al. Overall survival time prediction for high-grade glioma patients based on large-scale brain functional networks. Brain Imaging Behav. 2019;13(5): 1333-1351. https://doi.org/10.1007/s11682-018-9949-2

71. Dehkordi ANV, Kamali-Asl A, Wen N, Mikkelsen T, Chetty IJ, Bagher-Ebadian H. DCE-MRI prediction of survival time for patients with glioblastoma multiforme: using an adaptive neuro-fuzzy-based model and nested model selection technique. NMR Biomed. 2017;30(9). https://doi.org/10.1002/ nbm.3739

72. Smedley NF, El-Saden S, Hsu W. Discovering and interpreting transcriptomic drivers of imaging traits using neural networks. Bioinformatics. 2020;36(11):3537-48. https://doi.org/10.1093/ bioinformatics/btaal26

73. Chaddad A, Desrosiers C, Hassan L, Tanougast C. A quantitative study of shape descriptors from glioblastoma multiforme phenotypes for predicting survival outcome. Br J Radiol. 2016;89(1068):20160575. https://doi.org/10.1259/bjr.20160575

74. Zhang X, Lu D, Gao P, Tian Q, Lu H, Xu X, et al. Survival-relevant high-risk subregion identification for glioblastoma patients: the MRI-based multiple instance learning approach. Eur Radiol. 2020;30(10):5602-10. https://doi.org/10.1007/s00330-020-06912-8

75. Feng X, Tustison NJ, Patel SH, Meyer CH. Brain Tumor Segmentation Using an Ensemble of 3D U-Nets and Overall Survival Prediction Using Radiomic Features. Front Comput Neurosci. 2020;14:25. https://doi.org/10.3389/fncom.2020.00025

76. Zhuge Y, Krauze AV, Ning H, Cheng JY, Arora BC, Camphausen K, et al. Brain tumor segmentation using holistically nested neural networks in MRI images. Med Phys. 2017 Oct;44(10):5234-43. https://doi.org/10.1002/mp.12481

77. Sun L, Zhang S, Chen H, Luo L. Brain Tumor Segmentation and Survival Prediction Using Multimodal MRI Scans With Deep Learning. Front Neurosci. 2019;13:810. https://doi.org/10.3389/ fnins.2019.00810

78. Aquilanti E, Miller J, Santagata S, Cahill DP, Brastianos PK. Updates in prognostic markers for gliomas. Neuro-oncology. 2018;20(suppl 7):viil7-vii26. https://doi.org/10.1093/neuonc/noy158

79. Bell EH, Pugh SL, McElroy JP, Gilbert MR, Mehta M, Klimowicz AC, et al. Molecular-Based Recursive Partitioning Analysis Model for Glioblastoma in the Temozolomide Era: A Correlative Analysis Based on NRG Oncology RTOG 0525. JAMA Oncol. 2017;3(6):784-92.

80. Smail-Tabbone M, Rance B; Section Editors for the IMIA Yearbook Section on Bioinformatics and Translational Informatics. Contributions from the 2018 Literature on Bioinformatics and Translational Informatics. Yearb Med Inform. 2019;28(1):190-3. https://doi.org/10.1055/s-0039-1677945

81. Li M, Long S, Hu J, Wang Z, Geng C, Ou S. Systematic identification of lncRNA-based prognostic biomarkers for glioblastoma. Aging. 2019;11(21):9405-23. https://doi.org/10.18632/aging.102393

82. Qian Z, Li Y, Fan X, Zhang C, Wang Y, Jiang T, et al. Prognostic value of a microRNA signature as a novel biomarker in patients with lower-grade gliomas. J Neurooncol. 2018;137(1):127-37. https:// doi.org/10.1007/s11060-017-2704-5

83. Rosenberg S, Ducray F, Alentorn A, Dehais C, Elarouci N, Kamoun A, et al; POLA Network. Machine Learning for Better Prognostic Stratification and Driver Gene Identification Using Somatic Copy Number Variations in Anaplastic Oligodendroglioma. Oncologist. 2018;23(12):1500-10.

84. Petalidis LP, Oulas A, Backlund M, Wayland MT, Liu L, Plant K, et al. Improved grading and survival prediction of human astrocytic brain tumors by artificial neural network analysis of gene expression microarray data. Mol Cancer Ther. 2008;7(5):1013-24. https://doi.org/10.1158/1535-7163. MCT-07-0177

85. van der Voort SR, Incekara F, Wijnenga MMJ, Kapas G, Gardeniers M, Schouten JW, et al. Predicting the 1p/19q Codeletion Status of Presumed Low-Grade Glioma with an Externally Validated Machine 
Learning Algorithm. Clin Cancer Res. 2019;25(24):7455-62. https://doi.org/10.1158/1078-0432. CCR-19-1127

86. Han L, Kamdar MR. MRI to MGMT: predicting methylation status in glioblastoma patients using convolutional recurrent neural networks. Pac Symp Biocomput. 2018;23:331-42. https://doi. org/10.1142/9789813235533_0031

87. Kanemoto M, Shirahata M, Nakauma A, Nakanishi K, Taniguchi K, Kukita Y, et al. Prognostic prediction of glioblastoma by quantitative assessment of the methylation status of the entire MGMT promoter region. BMC Cancer. 2014;14:641. https://doi.org/10.1186/1471-2407-14-641

88. Nguyen HD, Allaire A, Diamandis P, Bisaillon M, Scott MS, Richer M. A machine learning analysis of a "normal-like" IDH-WT diffuse glioma transcriptomic subgroup associated with prolonged survival reveals novel immune and neurotransmitter-related actionable targets. BMC Med. 2020;18(1):280. https://doi.org/10.1186/s12916-020-01748-x

89. Saurabh R, Nandi S, Sinha N, Shukla M, Sarkar RR. Prediction of survival rate and effect of drugs on cancer patients with somatic mutations of genes: An AI-based approach. Chem Biol Drug Des. 2020;96(3):1005-19. https://doi.org/10.1111/cbdd.13668

90. Nalisnik M, Amgad M, Lee S, Halani SH, Velazquez Vega JE, Brat DJ, et al. Interactive phenotyping of large-scale histology imaging data with HistomicsML. Sci Rep. 2017;7(1):14588. https://doi. org/10.1038/s41598-017-15092-3

91. Powell RT, Olar A, Narang S, Rao G, Sulman E, Fuller GN, et al. Identification of Histological Correlates of Overall Survival in Lower Grade Gliomas Using a Bag-of-words Paradigm: A Preliminary Analysis Based on Hematoxylin \& Eosin Stained Slides from the Lower Grade Glioma Cohort of The Cancer Genome Atlas. J Pathol Inform. 2017;8:9. https://doi.org/10.4103/jpi.jpi_43_16

92. Senders JT, Staples P, Mehrtash A, Cote DJ, Taphoorn MJB, Reardon DA, et al. An Online Calculator for the Prediction of Survival in Glioblastoma Patients Using Classical Statistics and Machine Learning. Neurosurgery. 2020;86(2):E184-E192. https://doi.org/10.1093/neuros/nyz403 
